\title{
ON EXPONENTIAL STABILITY FOR LINEAR DIFFERENCE EQUATIONS WITH DELAYS
}

\author{
Leonid Berezansky1, brznsky@cs.bgu.ac.il, \\ Elena Braverman ${ }^{2}$ \\ ${ }^{1}$ Ben-Gurion University of the Negev, Beer-Sheva, Israel, \\ ${ }^{2}$ University of Calgary, Calgary, Canada
}

The article gives an overview of recent results on the stability of finite-difference equations with delay.

All results are compared with known signs of exponential stability of linear difference equations.

The results are obtained using the Bohl-Perron theorem and comparing the equation under study with an equation for which the Cauchy function is positive.

The Bohl-Perron theorem allows us to reduce the question of the exponential stability of a linear difference equation with delay to the solvability of an operator equation in one of the functional infinite-dimensional spaces.

That is, in fact, to an estimate of the norm or the spectral radius of a bounded linear operator in this space. For this estimation, different difference inequalities are used. One way to obtain such inequalities is to evaluate the fundamental solution in the event that this solution is positive.

The above scheme is used in this paper to obtain sufficient conditions for the exponential stability of the following equation

$x(n+1)-x(n)=-\sum_{l=1}^{m} a_{l}(n) x\left(h_{l}(n)\right), h_{l}(n) \leq n$,

provided that the coefficients and the lag are limited functions.

The main results of the paper are the following ones.

Theorem 1. Suppose the fundamental function of (1) is eventually positive, i.e., for some $n_{0} \geq 0$ we have $X(n, k)>0, n \geq k \geq n_{0}$, and, in addition,

$$
a=\lim _{n \rightarrow \infty} \text { inf } \sum_{l=1}^{m} a_{l}(n)>0 .
$$

Then equation (1) is exponentially stable.

Theorem 2. Suppose there exists a subset of indices $I \subset\{1,2, \ldots, m\}$ such that $a_{k} \geq 0, k \in I$, for the $\operatorname{sum} \sum_{l \in I} a_{l}(n)$ inequality (2) holds, the fundamental function $X_{1}(n, k)$ of the equation

$$
x(n+1)-x(n)+\sum_{l \in I} a_{l}(n) x\left(h_{l}(n)\right)=0
$$

is eventually positive and

$\lim _{n \rightarrow \infty} \sup \frac{\sum_{l \notin I}\left|a_{l}(n)\right|}{\sum_{l \in I} a_{l}(n)}<1$.

Then equation (1) is exponentially stable.

Keywords: linear difference equations, exponential stability, Bohl-Perron theorem, comparison theorems.

\section{Introduction}

In this paper we give a review on some new exponential stability results for scalar linear difference equations with bounded delays:

$$
x(n+1)-x(n)=-\sum_{l=1}^{m} a_{l}(n) x\left(h_{l}(n)\right), h_{l}(n) \leq n,
$$

where $h_{l}(n)$ is an integer for any $l=1, \ldots, m$ and $n=0,1,2, \ldots$ under the following two restrictions on the parameters of (1) which mean that coefficients and delays are bounded:

(a1) there exists $K>0$ such that $\left|a_{l}(n)\right| \leq K$ for $l=1, \ldots, m, n=0,1,2, \ldots$;

(a2) there exists $T>0$ such that $-T \leq h_{l}(n) \leq n$ for $l=1, \ldots, m, n=0,1,2, \ldots$.

To obtain explicit stability conditions we apply the following corollary of Bohl-Perron theorem. Denote by $\mathbf{I}^{\infty}$ the space of bounded sequences $v=\{x(n)\}$ with the norm $\|v\|_{I^{\infty}}=\sup _{n \geq 0}|x(n)|<\infty$.

Lemma 1 [2]. Suppose for the equation $\mathcal{L}(\{x(n)\})=0$, where 


$$
\mathcal{L}\left(\{x(n)\}_{n=0}^{\infty}\right)=\left\{x(n+1)-x(n)+\sum_{l=1}^{m} a_{l}(n) x\left(h_{l}(n)\right)\right\}_{n=0}^{\infty},
$$

conditions (a1)-(a2) hold, the equation $\mathcal{L}_{1}(\{x(n)\})=0$ is exponentially stable, where

$$
\mathcal{L}_{1}\left(\{x(n)\}_{n=0}^{\infty}\right)=\left\{x(n+1)-x(n)+\sum_{l=1}^{r} b_{l}(n) x\left(g_{l}(n)\right)\right\}_{n=0}^{\infty} .
$$

Let $Y(n, k)$ be the fundamental function of (2) and $C_{1}$ be the Cauchy operator of equation (2) which is

$$
C_{1}\{f(n)\}_{n=0}^{\infty}=\left\{\sum_{k=0}^{n-1} Y(n, k+1) f(k)\right\}_{n=0}^{\infty} .
$$

If the operator $T=C_{1}\left(\mathcal{L}_{1}-\mathcal{L}\right)=I-C_{1} \mathcal{L}$ satisfies $\|T\|_{I^{\infty} \rightarrow I^{\infty}}<1$, then the equation $\mathcal{L}(\{x(n)\})=0$ is exponentially stable.

By this lemma to obtain stability conditions we need to estimate norms of a some linear operator.

The problem is that this operator is given in an implicit form, since the fundamental function $Y(n, k)$ of a model equation usually is not known. So we can only estimate this function in some particular cases.

Such estimations we cam obtain for linear delay difference equations with positive fundamental functions.

\section{Equations with Positive fundamental Function}

The main results for such equations is the following.

Theorem 1. Suppose the fundamental function of (1) is eventually positive, i.e., for some $n_{0} \geq 0$ we have $X(n, k)>0, n \geq k \geq n_{0}$, and, in addition,

$$
a=\lim _{n \rightarrow \infty} \text { inf } \sum_{l=1}^{m} a_{l}(n)>0 .
$$

Then equation (1) is exponentially stable.

Further, we will apply nonoscillation tests, the following result is Theorems 4.1 in [3].

Lemma 2 [3]. Suppose $a_{l}(n) \geq 0, l=1,2, \ldots, m$, and for some $n_{0} \geq 0$

$$
\sup _{n \geq n_{0}} \sum_{l=1}^{m} a_{l}(n)<\frac{1}{2}, \sup _{n \geq n_{0}} \sum_{l=1}^{m} \sum_{k=\max \left\{n_{0}, \min _{l} h_{l}(n)\right\}}^{n-1} a_{l}(k) \leq \frac{1}{4} .
$$

Then the fundamental function of (1) is eventually positive: $X(n, k)>0, n \geq n_{0}$.

Corollary 1. Suppose $a_{l}(n) \geq 0$, (4) holds and (5) is satisfied for some $n_{0} \geq 0$. Then (1) is exponentially stable.

Consider together with (1) the following comparison equation

$$
x(n+1)-x(n)=-\sum_{l=1}^{m} b_{l}(n) x\left(g_{l}(n)\right), n \geq n_{0},
$$

where $g_{l}(n) \leq n$. Denote by $Y(n, k)$ the fundamental function of equation (6).

Lemma 3 [3]. Suppose $a_{l}(n) \geq b_{l}(n) \geq 0, g_{l}(n) \geq h_{l}(n), l=1,2, \ldots, m$, for sufficiently large n. If equation (1) has an eventually positive solution, then (6) has an eventually positive solution and its fundamental function $Y(n, k)$ is eventually positive.

Corollary 2. Suppose (4) and at least one of the following conditions hold:

1) $0 \leq a_{l}(n) \leq \alpha_{l}, h_{l}(n) \geq n-\tau_{l}$ and there exists $\lambda>0$ such that

$\lambda-1 \leq-\sum_{l=1}^{m} \alpha_{l} \lambda^{-\tau_{l}}$.

2) $m=1, n-h(n) \leq k ; a(n) \leq \frac{k^{k}}{(k+1)^{(k+1)}}$.

Then (1) is exponentially stable.

Remark 3. By Theorem 3.1 in [3] it is enough to assume the existence of an eventually positive solution in the conditions of Theorem 1 rather than to require that the fundamental function is positive.

\section{Explicit Stability Tests}

Further, we deduce explicit exponential stability conditions based on Lemmas 1 and 4. As above, we assume that (a1)-(a2) hold for (1).

Lemma 4. Suppose the fundamental function of the equation (1) is positive: $(n, k)>0, n \geq k \geq n_{0}$, and $a_{l}(n) \geq 0, l=1, \ldots, m, n \geq n_{0}$. Then there exists $n_{1} \geq n_{0}$ such that

$0 \leq \sum_{k=n_{0}}^{n-1} X(n, k+1) \sum_{l=1}^{m} a_{l}(k) \leq 1, n \geq n_{1}$.

Now let us proceed to explicit stability conditions. 
Theorem 2. Suppose there exists a subset of indices $I \subset\{1,2, \ldots, m\}$ such that $a_{k} \geq 0, k \in I$, for the sum $\sum_{l \in I} a_{l}(n)$ inequality (4) holds, the fundamental function $X_{1}(n, k)$ of the equation

$$
x(n+1)-x(n)+\sum_{l \in I} a_{l}(n) x\left(h_{l}(n)\right)=0
$$

is eventually positive and

$$
\lim _{n \rightarrow \infty} \sup \frac{\sum_{l \notin I}\left|a_{l}(n)\right|}{\sum_{l \in I} a_{l}(n)}<1 .
$$

Then equation (1) is exponentially stable.

Now let us proceed to explicit stability conditions.

Now we will take general exponentially stable difference equations with a positive fundamental function as a class of comparison equations.

Corollary 3. Suppose there exist a set of indices $I \subset\{1,2, \ldots, m\}$, functions $g_{l}(n) \leq n, l \in I$, and positive numbers $\alpha_{0}, \alpha_{1}$ and $\gamma<1$, such that for $n$ sufficiently large the inequalities

$$
0<\alpha_{0} \leq \sum_{l \in I} a_{l}(n) \leq \alpha_{1}<1, l \in I,
$$

hold and the difference equation

$$
x(n+1)-x(n)=-\sum_{l \in I} a_{l}(n) x\left(g_{l}(n)\right)
$$

has a positive fundamental function. If

$$
\sum_{k \in I}\left|a_{k}(n)\right| \sum_{j=\min \left\{h_{k}(n), g_{k}(n)\right\}}^{\max \left\{h_{k}(n), g_{k}(n)\right\}-1} \sum_{l=1}^{m}\left|a_{l}(j)\right|+\sum_{k \notin I}\left|a_{k}(n)\right| \leq \gamma \sum_{k \in I} a_{k}(n)
$$

then (1) is exponentially stable.

Remark 5. Based on the choice of subset $I$, the theorem contains $2^{m}-1$ different stability conditions.

Assuming $I=\{1,2, \ldots, m\}$ in Corollary 3 , we obtain the following result.

Corollary 4. Suppose there exists $g(n) \leq n$ and positive numbers $\alpha_{0}, \alpha_{1}$ and $\gamma<1$ such that for $n$ sufficiently large

$$
0<\alpha_{0} \leq b(n):=\sum_{l=1}^{m} a_{l}(n) \leq \alpha_{1}<1
$$

and the difference equation

$$
x(n+1)-x(n)=-\sum_{l=1}^{m} a_{l}(n) x(g(n))
$$

has a positive fundamental function. If for $n$ large enough

$$
\sum_{l=1}^{m}\left|a_{l}(n)\right| \sum_{k=\min \left\{h_{l}(n), g(n)\right\}}^{\max \left\{h_{l}(n), g(n)\right\}-1} \sum_{l=1}^{m}\left|a_{l}(k)\right| \leq \gamma \sum_{l=1}^{m} a_{l}(n),
$$

then equation (1) is exponentially stable.

The following result is an immediate corollary of Lemma 2 and Theorem 2.

Corollary 5. Suppose $0<a_{0} \leq a_{0}(n) \leq b_{0}<\frac{1}{4}$ and there exists $\gamma$ such that $0<\gamma<1$ and $\sum_{l=1}^{m}\left|a_{l}(n)\right| \leq \gamma a_{0}(n)$ for $n$ large enough. Then the equation

$$
x(n+1)-x(n)=-a_{0}(n) x(n-1)-\sum_{l=1}^{m} a_{l}(n) x\left(h_{l}(n)\right)
$$

is exponentially stable.

Corollary 6. Suppose for some positive $a_{0}, b_{0}, \gamma$, where $b_{0}<1, \gamma<1$, the following inequalities are satisfied for $n$ large enough

$$
\begin{aligned}
& 0<a_{0} \leq \sum_{l=1}^{m} a_{l}(n) \leq b_{0}<\frac{1}{4^{\prime}} \\
& \sum_{k=1}^{m}\left|a_{k}(n)\right| \sum_{j=h_{k}(n)}^{n-2} \sum_{l=1}^{m}\left|a_{k}(j)\right| \leq \gamma \sum_{l=1}^{m} a_{l}(n) .
\end{aligned}
$$

Then equation (1) is exponentially stable.

Now let us consider the case $m=2$

$x(n+1)-x(n)=-a(n) x(g(n))-b(n) x(h(n))$.

Corollary 7. Suppose delays $g(n), h(n)$ are bounded, there exist $a_{0}>0$ and $\gamma, 0<\gamma<1$ such that at least one of the following conditions holds for $n$ sufficiently large:

$$
\begin{gathered}
\text { 1) } 0<a_{0}<a(n) \leq a_{1}<\frac{1}{2}, \sum_{k=g(n)}^{n-1} a(k) \leq \frac{1}{4},|b(n)| \leq \gamma a(n) ; \\
\text { 2) } 0<a_{0} \leq a(n)+b(n) \leq a_{1}<\frac{1}{2}, \sum_{k=g(n)}^{n-1}(a(k)+b(k)) \leq \frac{1}{4} \text {, } \\
|a(n)| \sum_{\mathrm{k}=\min \{\mathrm{h}(\mathrm{n}), \mathrm{g}(\mathrm{n})\}}^{\max \{\mathrm{h}) \mathrm{g}(\mathrm{n})-1}[|a(k)|+|b(k)|]<\gamma[a(n)+b(n)] .
\end{gathered}
$$

Then equation (17) is exponentially stable. 
Proof. We choose the following equations:

$$
\begin{aligned}
& x(n+1)-x(n)=-a(n) x(g(n)), \\
& x(n+1)-x(n)=-a(n) x(g(n))-b(n) x(g(n)),
\end{aligned}
$$

with a positive fundamental function to obtain 1 ) and 2 ), respectively.

Consider now an autonomous equation with two delays

$x(n+1)-x(n)=-a x(n-g)-b x(n-h), a g \neq 0, b h \neq 0$.

Corollary 8. Suppose at least one of the following conditions holds:

1) $0<a \leq g^{g}(g+1)^{-(g+1)},|b|<a$;

2) $0<(a+b) \leq g^{g}(g+1)^{-(g+1)},|a(g-h)|<1$.

Then equation (18) is exponentially stable.

Consider a high order autonomous difference equation.

$x(n+1)-x(n)=-\sum_{l=1}^{m} a_{l} x(n-l)$.

Corollary 9. Suppose there exists $k \geq 1$ such that

$$
0<\sum_{l=1}^{k} a_{l} \leq \frac{k^{k}}{(k+1)^{(k+1)}}, \sum_{l=k+1}^{m}\left|a_{l}\right|<\sum_{l=1}^{k} a_{l} .
$$

Then equation (19) is exponentially stable.

\section{Discussion and Examples}

Let us note that the approach using Bohl-Perron Theorem is similar to the method developed in [20] where stability is deduced based on the fact that some linear exponentially stable equation is close to the considered equation. Unlike the present paper, [20] considers nonlinear perturbations of stable linear equations as well. The main result (Theorem 2) of [20] is the following one.

Suppose that the fundamental function of (1) satisfies

$$
\sum_{j=0}^{n-1}|X(n, j+1)| \leq L, n=n_{0}, n_{0}+1, \ldots .
$$

Then the nonlinear equation

$$
x(n+1)-x(n)=-\sum_{k=1}^{m} a_{k}(n) x\left(h_{k}(n)\right)+F(n, x(n), x(n-1), \ldots, x(n-l))
$$

is globally asymptotically stable if in addition $\mid F\left(n, x_{0}, x_{1}, \ldots, x_{l}\left|\leq q \max _{0 \leq i \leq l}\right| x_{i} \mid\right.$, and $q<L^{-1}$.

Instead of inequality (20) in this paper we apply exponential estimation

$\mid X\left(n, k \mid \leq M e^{-\gamma(n-k)}, M>0, \gamma>0\right.$.

Generally, (21) implies (20), however for bounded delays and coefficients

Let us discuss some stability tests for equation (1).

We start with the following result $[9,19,23,24]$.

If $m=1, \sum_{n=0}^{\infty} a(n)=\infty, n-h(n) \leq k, a(n) \geq 0$, and

$\sum_{i=h(n)}^{n} a(i)<\frac{3}{2}+\frac{1}{2 k+2}$

then equation (1) is asymptotically stable. This result is also true for general equation (1) ( $m>1)$, where $a_{l}(n) \geq 0, a(n)=\sum_{l=1}^{n} a_{l}(n), h(n)=\max h_{l}(n)$.

Equation (1) with positive constant coefficients is asymptotically stable if [10]

$$
\sum_{l=1}^{m} a_{l} \lim _{n \rightarrow \infty} \sup \left(n-h_{l}(n)\right)<1+\frac{1}{e}-\sum_{l=1}^{m} a_{l} .
$$

Stability tests (22) and (23) are obtained for equations with positive coefficients. In the present paper we consider coefficients of arbitrary signs. The next interesting feature of the results obtained here is that some of the delays can be arbitrarily large (see for example, Parts 1 and 2 of Corollary 7).

Example 1. By Corollary 7, Part 1, the following two equations

$$
\begin{aligned}
& x(n+1)-x(n)=-(0,2+0,05 \sin \mathrm{n}) x(n-1)-0,1|\cos \mathrm{n}| x(n-20), \\
& x(n+1)-x(n)=-\left[0,12+0,1(-1)^{n}\right] x(n-2)-\left[0,1+0,11(-1)^{n}\right] x(n-14)
\end{aligned}
$$

are exponentially stable.

Two previous results of [9, 19, 23, 24] and [10] fail to establish exponential stability for equation (24) with positive coefficients, as well as all parts of Corollary 3.10 in [4] cannot be applied to equation 
(25) with an oscillating coefficient. None of the inequalities in Corollary 8 of [2] can be applied to establish stability of (25).

We note that (24), (25) are special cases of equation with one nondelay term and two delay terms considered in [2], however none of the inequalities in Corollary 8 of [2] can be applied to establish stability of (25). Let us also note that for (25) we have

$$
\sum_{k=g(n)}^{n-1}|a(k)|+\sum_{j=h(n)}^{n-1}|b(j)|=1.78>\frac{\pi}{2}
$$

where [13] $\pi / 2$ is the best possible constant [19, 22] in $\sum_{k=1}^{m} k a_{k}<\frac{\pi}{2}$ which implies exponential stability of (19).

Example 2. Consider equation (17) with two variable coefficients and delays, where

$$
\begin{aligned}
& a(n)=\left\{\begin{array}{l}
-0.12, \text { if } n \text { is even, } \\
-0.05, \text { if } n \text { is odd },
\end{array} \quad b(n)=\left\{\begin{array}{l}
0.17, \text { if } n \text { is even, } \\
0.08, \text { if } n \text { is odd, }
\end{array}\right.\right. \\
& g(n)=\left\{\begin{array}{l}
n-3, \text { if } n \text { is even, } \\
n-5, \text { if } n \text { is odd, }
\end{array} h(n)=\left\{\begin{array}{l}
n-4, \text { if } n \text { is even, } \\
n-8, \text { if } n \text { is odd, }
\end{array}\right.\right.
\end{aligned}
$$

Then in (17) the sum $a(n)+b(n)$ is either 0.05 or 0.03 which is less than 0.5 ,

$$
\sum_{k=n-5}^{n-1}[a(k)+b(k)] \leq 3 \cdot 0.05+2 \cdot 0.03=0.21<1 / 4
$$

for both odd and even $n$ and for $\gamma=0.95<1$ we have

$$
|a(n)| \sum_{k=\min \{h(n), g(n)\}}^{\max \{h(n), g(n)\}-1}[|a(k)|+|b(k)|]=\left\{\begin{array}{c}
0.0348<0.05 \gamma, n \text { is even, } \\
0.0275<0.03 \gamma, n \text { is odd, }
\end{array}\right.
$$

thus by Corollary 7, Part 3, equation (17) is exponentially stable. None of the inequalities in Corollary 8 of [2] can be applied to establish stability of (17). We note that it would be harder to treat this example if the equation were written as high order equations with constant delays and variable coefficients.

A number of papers $[2,12,14,15,16,17,20]$ are devoted to stability tests for equations with positive and negative coefficients and, more generally, for equations with oscillating coefficients. Paper [20] extends earlier results of [12]. In particular, for the linear autonomous equation

$$
x(n+1)-x(n)=q x(n-m)-p x(n-k), p>0, q>0, m \geq 1, k \geq 1 .
$$

the following result was obtained in [12].

Suppose $p \frac{(k+1)^{(k+1)}}{k^{k}} \leq 1$. Then equation(28) is exponentially stable if and only if $p>q$.

Condition 1) of Corollary 8 is close to this result. It gives the same sufficient stability test for $q$ of an arbitrary sign but does not involve the necessity part.

The paper [16] contains a nice review on stability results obtained for equations with oscillating coefficients. The results of [16] generalized the following stability test obtained in [14] for equation (28):

If $k p<1, p \frac{1-k p}{1+k p}>q$ then (28) is asymptotically stable.

By condition 2) of Corollary 8 equation (28) is asymptotically stable if

$$
p-q<\frac{k^{k}}{(k+1)^{(k+1)}},|p(k-m)|<1 .
$$

It is easy to see that these two tests are independent.

Let us discuss sharpness of conditions of Theorem 1 for exponential stability of (1), assuming the fundamental function is positive; in particular, we demonstrate sharpness of condition (4).

Example 3. The equation

$$
x(n+1)-x(n)=-3^{-n-1} x(n), n \geq n_{0} \geq 0,
$$

has a positive fundamental function and any solution can be presented as $x(n)=x\left(n_{0}\right) \prod_{k=n_{0}}^{n-1}\left(1-3^{-k-1}\right)$, thus for $n_{0}=0$

$$
X(n, 0)=\prod_{k=0}^{n-1}\left(1-3^{-k-1}\right)>1-\sum_{k=0}^{n-1} 3^{-k-1}>1-\sum_{k=0}^{\infty} 3^{-k-1}=\frac{1}{2}
$$

i.e., the equation is neither asymptotically nor exponentially stable.

Let us demonstrate that the facts that the sum of coefficients $\sum_{l=1}^{m} a_{l}(n)$ in (1) is positive, exceeds a positive number and that the fundamental function is positive do not imply stability, in the case when coefficients have different signs. 
Example 4. Consider the difference equation

$x(n+1)-x(n)=-2.2 x(n-1)+2 x(n)$.

Here $2.2-2=0.2>0$, so the sum of coefficients exceeds a certain positive number. Let us prove that the fundamental function is positive and the solution is unbounded. Really, for the fundamental function we have $X(0,0)=1, X(1,0)=3$. Denote $x(n)=X(n, 0)$, notice that $x(1)>1,5 x(0)$ and prove $x(n)>1,5 x(n-1) 0$ by induction. Really, $x(n)>1,5 x(n-1)>0$ implies $x(n-1)<2 x(n) / 3$, and for any $x(n-1)>0$ we have

$$
x(n+1)=3 x(n)-2.2 x(n-1)>3 x(n)-\frac{4.4 x(n)}{3}=\frac{4.6}{3} x(n)>1.5 x(n),
$$

thus $X(n, 0)$ is positive and unbounded. The equation is autonomous, so the same is true for $X(n, k)$. Since $X(n, 0)$ is unbounded, then (29) is not stable.

Finally, let us formulate some open problems.

1. Under which conditions will exponential stability of (1) imply exponential stability of the equation with the same coefficients and smaller delays:

$x(n+1)-x(n)=-\sum_{l=1}^{m} a_{l}(n) x\left(g_{l}(n)\right), n \geq n_{0}, h_{l}(n) \leq g_{l}(n) \leq n$.

2. Prove or disprove:

If in Theorem 2 condition (10) is substituted by

$\sum_{l \notin I}\left|a_{l}(n)\right| \leq \alpha_{n} \sum_{l \in I} a_{l}(n), \prod_{n=1}^{\infty} \alpha_{n} \leq 1$

and all other assumptions hold, then (1) is stable. If in addition

$\sum_{n=1}^{\infty}\left(1-\alpha_{n}\right)=\infty$,

then (1) is asymptotically stable.

3. Consider the problem of the exponential stability of (1) when (a1)-(a2) are substituted with one of two more general conditions:

a) $\lim _{n \rightarrow \infty} h_{l}(n)=\infty$ and there exists $M>0$ such that

$\sum_{j=h_{l}(n)}^{n} a_{l}(j)<M$, for any $l=1, \ldots, m$;

b) delays are infinite but coefficients decay exponentially with memory, i.e., there exist positive numbers $M$ and $\lambda<1$ such that $\left|a_{l}(n)\right| \leq M \lambda^{n-h_{l}(n)}$.

Let us note that Bohl-Perron type result in case b) was obtained in [5], Theorem 4.7.

4. Example 5 demonstrates that for equations with positive and negative coefficients and a positive fundamental function inequality (4) does not imply exponential stability. Is it possible to find such conditions on delays and coefficients of different signs that (4) would imply exponential stability? For instance, prove or disprove the following conjecture.

Conjecture. Suppose the following conditions

$a(n) \geq b(n) \geq 0, h(n) \leq g(n) \leq n, \lim _{n \rightarrow \infty} \sup b(n)[g(n)-h(n)]<1$

are satisfied for the equation

$$
x(n+1)-x(n)=-a(n) x(h(n))+b(n) x(g(n)) .
$$

If the fundamental function of (30) is positive and

$\lim _{n \rightarrow \infty} \inf [a(n)-b(n)]>0$,

then (30) is exponentially stable.

Let us remark that conditions when the fundamental function of (30) is positive were obtained in [6].

\section{References}

1. Berezansky L., Braverman E. On Bohl-Perron Type Ttheorems for Linear Difference Equations. Funct. Differ. Equ., 2004, vol. 11, no. 1-2, pp. 19-28.

2. Berezansky L., Braverman E. On Exponential Dichotomy, Bohl-Perron Type Theorems and Stability of Difference Equations. J. Math. Anal. Appl., 2005, vol. 304, pp. 511-530. DOI: 10.1016/j.jmaa.2004.09.042

3. Berezansky L., Braverman E. On Existence of Positive Solutions for Linear Difference Equations with Several Delays. Adv. Dyn. Syst. Appl., 2006, vol. 1, no. 1, pp. 29-47. 
4. Berezansky L., Braverman E., Liz E. Sufficient Conditions for the Global Stability of Nonautonomous Higher Order Difference Equations. J. Difference Equ. Appl., 2005, vol. 11, no. 9, pp. 785-798. DOI: 10.1080/10236190500141050

5. Berezansky L., Braverman E. On Exponential Dichotomy for Linear Difference Equations with Bounded and Unbounded Delay. Proceedings of the Conference on Differential and Difference Equations \& Applications Melbourne, Florida, August 1-5, 2005. Edited by Ravi P. Agarwal and Kanishka Perera, Hindawi Publishing Corporation, 2006, pp. 169-178.

6. Berezansky L., Braverman E., Kravets O. Nonoscillation of Linear Delay Difference Equations with Positive and Negative Coefficients. J. Difference Equ. Appl., 2008, vol. 14, no. 5, pp. 495-511. DOI: $10.1080 / 10236190701692273$

7. Elaydi S., Periodicity and Stability of Linear Volterra Difference Systems. J. Math. Anal. Appl., 1994, vol. 181, no. 2, pp. 483-492. DOI: 10.1006/jmaa.1994.1037

8. Elaydi S. and Murakami S. Uniform Asymptotic Stability in Linear Volterra Difference Equations. J. Difference Equ. Appl., 1998, vol. 3, no. 3-4, pp. 203-218. DOI: 10.1080/10236199808808097

9. Erbe L.H., Xia H., Yu J.S., Global Stability of a Linear Nonautonomous Delay Difference Equation. J. Difference Equ. Appl., 1995, vol. 1, no. 2, pp. 151-161. DOI: 10.1080/10236199508808016

10. Györi I., Hartung F. Stability in Delay Perturbed Differential and Difference Equations. Fields Inst. Commun., 2001, vol. 29, pp. 181-194. DOI: 10.1090/fic/029/14

11. Györi I. and Ladas G. Oscillation Theory of Delay Differential Equations. Clarendon Press, Oxford, $1991.378 \mathrm{p}$.

12. Györi I., Ladas G., Vlahos P.N. Global Attractivity in a Delay Difference Equation. Nonlinear Anal. TMA, 1991, vol. 17, no. 5, pp. 473-479. DOI: 10.1016/0362-546x(91)90142-n

13. Kipnis M.M., Komissarova D.A. A Note of Explicit Stability Conditions of Autonomous Higher Order Difference Equation. J. Difference Equ. Appl., 2007, vol. 13, no. 5, pp. 457-461. DOI: $10.1080 / 10236190601132933$

14. Kocić V.L., Ladas G. Global Behavior of Nonlinear Difference Equations of Higher Order with Applications. Math. Appl,, vol. 256. Kluwer Academic, Dordrecht, 1993. 240 p. DOI: 10.1007/978-94-017-1703-8

15. Krause U., Pituk M. Boundedness and Stability for Higher Order Difference Equations. J. Differ. Equations Appl., 2004, vol. 10, pp. 343-356. DOI: 10.1080/1023619031000115377

16. Liz E. On Explicit Conditions for the Asymptotic Stability of Linear Higher Order Difference Equations. J. Math. Anal. Appl., 2005, vol. 303, pp. 492-498. DOI: 10.1016/j.jmaa.2004.08.048

17. Liz E., Ivanov A., Ferreiro J.B., Discrete Halanay-Type Inequalities and Applications. Nonlinear Anal., 2003, vol. 55, pp. 669-678. DOI: 10.1016/j.na.2003.07.013

18. Liz E., Pituk M. Asymptotic Estimates and Exponential Stability for Higher-Order Monotone Difference Equations. Adv. Difference Equ., 2005, no. 1, pp. 41-55. DOI: 10.1155/ADE.2005.41

19. Malygina V.V., Kulikov A.Y. On Precision of Constants in Some Theorems on Stability of Difference Equations. Func. Differ. Equ., 2008, vol. 15, no. 3-4, pp. 239-249.

20. Pituk M. Global Asymptotic Stability in a Perturbed Higher Order Linear Difference Rquation, Comput. Math. Appl., 2003, vol. 45, pp. 1195-1202. DOI: 10.1016/S0898-1221(03)00084-1

21. Tang X.H., Jiang Z. Asymptotic Behavior of Volterra Difference Equation. J. Difference Equ. Appl., 2007, vol. 13, no. 1, pp. 25-40. DOI: 10.1080/10236190601008810

22. Tkachenko V. and Trofimchuk S. A Global Attractivity Criterion for Nonlinear NonAutonomous Difference Equations. J. Math. Anal. Appl., 2006, vol. 322, no. 2, pp. 901-912. DOI: 10.1016/j.jmaa.2005.09.052

23. Yu J.S. Asymptotic Stability for a Linear Difference Equation with Variable Delay, Advances in Difference Equations, II. Comput. Math. Appl. 36, 1998, no. 10-12, pp. 203-210. DOI: 10.1016/s0898-1221(98)80021-7

24. Zhang B.G., Tian C.J., Wong P.J.Y. Global Attractivity of Difference Equations with Variable Delay. Dynam. Contin. Discrete Impuls. Systems, 1999, vol. 6, no. 3, pp. 307-317.

Received 31 May 2018 


\title{
ОБ ЭКСПОНЕНЦИАЛЬНОЙ УСТОЙЧИВОСТИ ЛИНЕЙНЫХ РАЗНОСТНЫХ УРАВНЕНИЙ С ЗАДЕРЖКАМИ
}

\author{
Л. Березанский ${ }^{1}$, Е. Браверман ${ }^{2}$ \\ ${ }^{1}$ Университет им. Бен-Гуриона в Негеве, г. Беэр-Шева, Израиль, \\ ${ }^{2}$ Университет Калгари, г. Калгари, Канада
}

Приведен обзор последних результатов по устойчивости разностных уравнений с запаздыванием.

Все результаты сравниваются с известными признаками экспоненциальной устойчивости линейных разностных уравнений.

Результаты получены с использованием теоремы Боля - Перрона и сравнения исследуемого уравнения с уравнением, у которого функция Коши положительна.

Теорема Боля - Перрона позволяет вопрос об экспоненциальной устойчивости линейного разностного уравнения с запаздыванием сводить к разрешимости операторного уравнения в одном из функциональных бесконечномерных пространств.

То есть фактически к оценке нормы или спектрального радиуса линейного ограниченного оператора в этом пространстве. Для такой оценки используются различные разностные неравенства. Один из способов получения таких неравенств заключается в оценке фундаментального решения в том случае если это решение положительно.

Изложенная выше схема применяется в данной работе для получения достаточных условий экспоненциальной устойчивости следующего уравнения

$x(n+1)-x(n)=-\sum_{l=1}^{m} a_{l}(n) x\left(h_{l}(n)\right), h_{l}(n) \leq n$,

при условии, что коэффициенты и запаздывания - ограниченные функции.

Основными результатами работы являются следующие.

Теорема 1. Пусть фундаментальная функция (1) в конечном итоге положительна, т. е. для некоторого $n_{0} \geq 0$ имеем $X(n, k)>0, n \geq k \geq n_{0}$ и, кроме того,

$a=\lim _{n \rightarrow \infty}$ inf $\sum_{l=1}^{m} a_{l}(n)>0$.

Тогда уравнение (1) экспоненциально устойчиво.

Теорема 2. Пусть существует подмножество индексов $I \subset\{1,2, \ldots, m\}$ таких, что $a_{k} \geq 0$, $k \in I$, для суммы $\sum_{l \in I} a_{l}(n)$ выполнено неравенство (2), фундаментальные функции $X_{1}(n, k)$ уравнения

$$
x(n+1)-x(n)+\sum_{l \in I} a_{l}(n) x\left(h_{l}(n)\right)=0
$$

в конечном итоге положительные и

$\lim _{n \rightarrow \infty} \sup \frac{\sum_{l \notin I}\left|a_{l}(n)\right|}{\sum_{l \in I} a_{l}(n)}<1$.

Тогда уравнение (1) экспоненциально устойчиво.

Ключевые слова: линейные разностные уравнения, экспоненциальная устойчивость, теорема Боля - Перрона, теоремы сравнения.

Березанский Леонид, департамент математики, Университет им. Бен-Гуриона в Негеве, г. Беэр-Шева, Израиль; brznsky@cs.bgu.ac.il.

Браверман Елена, департамент математики и статистики, Университет Калгари, г. Калгари, Канада.

Поступила в редакцию 31 мая 2018 2.

\section{ОБРАЗЕЦ ЦИТИРОВАНИЯ}

Berezansky, L. On Exponential Stability for Linear Difference Equations with Delays / L. Berezansky, E. Braverman // Вестник ЮУрГУ. Серия «Компьютерные технологии, управление, радиоэлектроника». - 2018. T. 18, № 3. - C. 31-38. DOI: 10.14529/ctcr180304

\section{FOR CITATION}

Berezansky L., Braverman E. On Exponential Stability for Linear Difference Equations with Delays. Bulletin of the South Ural State University. Ser. Computer Technologies, Automatic Control, Radio Electronics, 2018, vol. 18, no. 3, pp. 31-38. DOI: 10.14529/ctcr180304 